\title{
I CANDIDATI
}

\author{
di Liborio Mattina
}

Le valutazioni sui mutamenti determinati dalle elezioni politiche del marzo 1994 sono contrastanti. Molta pubblicistica politica ha affermato che le elezioni hanno provocato un profondo cambiamento nella composizione del ceto politico parlamentare, altri hanno sostenuto, invece, che era avvenuta solo un'operazione di maquillage che ha portato $\mathrm{i}$ «ragazzi del coro» in prima fila a cogliere la grande occasione offerta dal forzato ritiro dei leader identificati con l'odiata partitocrazia. Uno degli obiettivi di questo saggio è dimostrare che entrambe le interpretazioni sono sbagliate, la prima perché identifica il rinnovamento semplicemente con il cambio delle «facce» mentre esso va valutato in relazione alla composizione sociologica e alle caratteristiche professionali del nuovo ceto parlamentare; l'altra perché sopravvaluta le possibilità delle élite politiche di continuare a premiare ad libitum se stesse ed i fedeli, trascurando di contribuire al rinnovamento del personale politico in un periodo di transizione politico-istituzionale contrassegnato da una notevole crisi di legittimazione delle burocrazie partitiche.

L'analisi delle candidature presentate nelle diverse liste elettorali confermerà che i partiti - vecchi e nuovi - sono riusciti a mantenere il controllo dei canali di selezione del ceto parlamentare, anche perché è sostanzialmente mancata l'attivazione di canali complementari o alternativi di reclutamento, quali le associazioni di rappresentanza dei grandi interessi economici. D'altra parte, verrà mostrato che i partiti sono stati indotti ad aprire le liste agli ingressi esterni in misura significativa e a contenere la presenza dei politici di professione. Illustreremo, inol-

\footnotetext{
Ringrazio Daniele Nania per la preziosa assistenza prestata durante la elaborazione informatica dei dati.
}

RIVISTA ITALIANA DI SCIENZA POLITICA / a. XXIV, n. 3, dicembre 1994 
tre, come hanno potuto coesistere le modalità di selezione più tradizionali con quelle più aperte a raccogliere le candidature offerte da ceti sociali privi di esperienze politiche precedenti a quella della candidatura. L'analisi sulle candidature verrà effettuata ponendo a confronto le strategie di reclutamento adottate dalle diverse coalizioni politiche e dalle liste ad esse collegate.

\section{Il campione, l'età ed il reddito dei candidati}

Il nostro campione è costituito da 1204 candidati $(89 \%$ uomini e $11 \%$ donne) nei collegi uninominali istituiti con il sistema maggioritario per l'elezione dei tre quarti dei componenti della Camera e del Senato. In particolare, 794 si sono presentati in 243 dei 475 collegi uninominali per le elezioni alla Camera e 410 in 131 dei 232 collegi uninominali istituiti per l'elezione dei senatori. Complessivamente $\mathrm{i}$ candidati del campione, tra elezioni per la Camera e il Senato, si sono presentati in 374 collegi uninominali, cioè nel 52,8\% dei 707 collegi uninominali istituiti con il nuovo sistema elettorale.

I 1204 del campione rappresentano il $33,2 \%$ di tutti i 3622 candidati presentatisi nei collegi elettorali uninominali per la Camera e il Senato. C'è, però, da aggiungere che con il nostro campione esaminiamo solo i candidati presentati dalla coalizione dei Progressisti (Pgr), dal Patto per l'Italia, dal Polo delle Libertà e dal Polo del buon governo (Pdl-Pbg), e delle liste ad esse collegate per la conquista dei seggi attribuiti alla quota destinata al recupero proporzionale. Perciò il nostro campione rappresenta oltre il $40 \%$ del totale dei candidati presentatisi nelle tre coalizioni elettorali maggiori.

Le informazioni sui candidati derivano dai sintetici profili biografici pubblicati nelle settimane precedenti alle elezioni sulle pagine locali dei quotidiani. I profili sono stati realizzati con criteri diversi. Di ciò ha risentito la qualità e la completezza delle informazioni raccolte. Pur nei limiti delle fonti utilizzate il nostro obiettivo è stato di acquisire dati affidabili sui candidati relativi a: età, reddito, coalizione politica di appartenenza, lista elettorale di collegamento nella quota proporzionale, attività professionale svolta, eventuali cariche direttive assunte in parti$\mathrm{ti}$, organizzazioni di interesse $\mathrm{o}$ associazioni volontarie, eventuali precedenti esperienze parlamentari o in assemblee elettive locali. Raramente è stato possibile acquisire informazioni esaurienti 
su tutti questi elementi. Tuttavia sono stati inseriti nel nostro campione tutti i candidati per i quali è stato possibile acquisire almeno un'informazione importante sul loro curriculum politico professionale, oltre a quelle relative alla coalizione politica che lo ha presentato. Inevitabilmente, la disomogeneità delle informazioni ha frequentemente imposto di limitare l'analisi sulle caratteristiche dei candidati ad un numero più ristretto di quello complessivo dei soggetti considerati nel campione.

Il campione dei candidati è stato selezionato in collegi compresi in 16 differenti circoscrizioni per la Camera e in 8 per il Senato con l'intento di renderlo rappresentativo delle tre aree territoriali, abbastanza omogenee per caratteristiche socio-economiche ed orientamenti di voto, in cui si può suddividere il paese (Tab. 1).

Tra le singole liste che fanno parte delle tre coalizioni considerate nel campione il numero dei candidati di Alleanza Nazionale (An) appare sovradimensionato rispetto a quello delle altre due formazioni maggiori, Forza Italia (Fi) e Lega nord ( $\mathrm{Ln}$ ), che hanno dato vita alla coalizione di destra. Ciò è accaduto perché An al Nord e al Centro ha presentato candidati concor-

ТАВ. 1. Il numero di candidati del campione distinto per coalizione nelle aree prescelte

\begin{tabular}{lccccc}
\hline AREA & \multicolumn{3}{c}{ COALIZIONI } & & \\
\cline { 2 - 4 } & Pgr & PpI & Pdl-Pbg & Tot. & $(\%)$ \\
\hline $\begin{array}{l}\text { Piemonte } \\
\text { Lombardia } \\
\begin{array}{l}\text { Veneto } \\
\text { NORD }\end{array}\end{array}$ & 127 & 128 & 268 & 523 & $(43,3)$ \\
$\begin{array}{l}\text { Emilia } \\
\text { e Romagna }\end{array}$ & & & & & \\
$\begin{array}{l}\text { Toscana } \\
\text { CENTRO }\end{array}$ & 65 & 63 & 115 & 243 & $(20,3)$ \\
$\begin{array}{l}\text { Lazio } \\
\text { Campania } \\
\text { Sicilia } \\
\text { SUD }\end{array}$ & & & & & \\
Totale & 152 & 134 & 152 & 438 & $(36,4)$ \\
\hline
\end{tabular}

Legenda $:$ Pgr $=$ Progressisti; $\mathrm{PpI}=$ Patto per l'Italia; $\mathrm{Pdl}-\mathrm{Pbg}=$ Polo delle libertà + Polo del buon governo. 
renti con quelli del Polo delle libertà, e in alcuni collegi campa$\mathrm{ni}$ in competizione con quelli di Fi. Sul versante della coalizione di sinistra abbiamo sempre mantenuto presente la differente appartenenza di lista dei candidati, ma abbiamo utilizzato solo quelli del $\mathrm{Pds}$ per effettuare il confronto tra i candidati delle più importanti liste dei tre schieramenti. Infine, una certa difficoltà riscontrata nella fase di rilevazione dei dati nel distinguere tra i candidati collegati al Patto Segni e quelli del Partito popolare all'interno del Patto per l'Italia, ci ha indotto a considerare come appartenenti ad un'unica lista i candidati delle due distinte formazioni.

Un primo e molto generale indicatore dell'eventuale cambiamento nel reclutamento dei candidati per le cariche elettive è dato dal ricambio generazionale e dal grado di rappresentatività sociale dei candidati rispetto agli elettori. Quest'ultima può essere misurata con numerosi indicatori. Uno dei più semplici è il reddito individuale. Ebbene, l'età e il reddito dei candidati del nostro campione offrono diverse conferme alle ipotesi ed anticipazioni formulate durante la campagna elettorale.

Riguardo all'età emerge con chiarezza che, nonostante gli

TAB. 2. L'età media dei candidati del campione (\%)

\begin{tabular}{lccc}
\hline & Camera & Senato & (n. casi: 722) \\
\hline Pds & 46,4 & 50,6 & $(108)$ \\
PpI & 47,4 & 53,4 & $(257)$ \\
$\mathrm{Ln}$ & 43,2 & 51,4 & $(85)$ \\
$\mathrm{Fi}$ & 45,1 & 54,3 & $(84)$ \\
$\mathrm{An}$ & 46,9 & 56,3 & $(188)$ \\
\hline
\end{tabular}

Le fasce d'età

\begin{tabular}{lccccccl}
\hline & Pds & PpI & Ln & Fi & An & Totale & \\
\hline $27-35$ anni & 4,6 & 7,4 & 22,4 & 12 & 11,2 & 11,8 & $(55)$ \\
$36-45$ & 37 & 24,5 & 23,5 & 29,8 & 29,8 & 30,3 & $(141)$ \\
$46-55$ & 44,5 & 44,7 & 37,7 & 36,9 & 26 & 34,4 & $(160)$ \\
$56-65$ & 10,2 & 18,7 & 12,9 & 15,4 & 18,1 & 14,8 & $(69)$ \\
oltre i 65 & 3,7 & 4,7 & 3,5 & 5,9 & 14,9 & 8,7 & $(40)$ \\
Totale & 100 & 100 & 100 & 100 & 100 & 100 & \\
(N) & $(108)$ & $(257)$ & $(85)$ & $(84)$ & $(188)$ & & $(722)$ \\
\hline
\end{tabular}


sforzi compiuti, testimoniati dal ritiro volontario, o imposto, di quasi la metà dei deputati e senatori della XI legislatura, i partiti politici sono riusciti a proporre solo un moderato ricambio generazionale nel Parlamento. Infatti, l'età media dei candidati delle diverse liste si colloca (Tab. 2) al di sotto della media generale dei deputati nelle ultime due legislature (che era di 49,5 anni), ma è più elevata dell'età media della prima elezione (che, ancora nelle ultime due legislature, era di 45 anni) (Censis 1994). Fanno eccezione a questa tendenza i candidati di Fi e della Ln. Quest'ultima si conferma, come era già emerso nella XI legislatura, la formazione politica più giovane: quasi il $46 \%$ dei suoi candidati sono compresi nelle due fasce d'età più basse; in particolare in quella tra i 27 e i 35 anni la Lega ha un numero di candidati almeno doppio (con l'eccezione di Fi) di quello delle altre formazioni politiche. I partiti politici con una presenza parlamentare più strutturata hanno invece puntato soprattutto sulla fascia d'età centrale (46-55 anni), con l'eccezione rilevante di An. Il $33 \%$ dei candidati di questa formazione si colloca nelle due fasce d'età più elevate. Del resto questa formazione ha confermato quasi tutti i parlamentari eletti nelle file dell'Msi nell'XI legislatura, contribuendo, in tal modo, a elevare l'età media dei suoi candidati.

I dati sul reddito dei candidati vanno presi con beneficio d'inventario perché il numero di casi relativo ad alcune liste è molto limitato ed anche perché è nota la scarsa inclinazione del contribuente italiano, ancorché candidato al Parlamento, ad essere esplicito su tale argomento. Pur adottando le cautele dovute alle possibili distorsioni provocate dalla natura dell'argomento e dal limitato numero di casi, non si può fare a meno di no-

TAB. 3. Il reddito dei candidati in milioni di lire (lordo 1992) (\%)

\begin{tabular}{lccrrrr}
\hline Classi di reddito & Pds & \multicolumn{1}{c}{ PpI } & Fi & Ln & An & Tot. \\
\hline Fino a 34 & 18,2 & 8,3 & 12,5 & 18,9 & 24,7 & 20,1 \\
$35-50$ & 13,7 & 21,3 & 9,4 & 13,5 & 18,5 & 14,9 \\
$51-75$ & 9,1 & 15,7 & 3,1 & 21,6 & 18,5 & 14,4 \\
$76-100$ & 20,6 & 13,9 & 21,8 & 21,6 & 13,6 & 18,1 \\
$101-150$ & 29,3 & 27,8 & 18,8 & 16,3 & 16,1 & 19,6 \\
oltre i 150 & 9,1 & 13 & 34,4 & 8,1 & 8,6 & 12,9 \\
& & & & & & \\
Totale & 100 & 100 & 100 & 100 & 100 & 100 \\
(N) & $(44)$ & $(108)$ & $(32)$ & $(37)$ & $(81)$ & $(302)$ \\
\hline
\end{tabular}


tare che la tabella 3 conferma una delle previsioni della vigilia delle elezioni e cioè che in Fi prevalgono i candidati che provengono dai settori sociali economicamente più elevati: oltre il $53 \%$ dei candidati di $\mathrm{Fi}$ ha avuto nel 1992 un reddito annuo superiore ai 100 milioni. I candidati di Fi sono seguiti, oltre la soglia dei 100 milioni, ma a molta distanza, da quelli del Pds (il $38,4 \%$ ).

Strategie di reclutamento

Entriamo, ora, più nel merito della nostra analisi cercando di identificare quali sono stati i criteri di reclutamento adottati dai partiti.

In linea di principio i partiti dovrebbero selezionare candidati in grado di rappresentare gli interessi della nazione conciliando le aspettative della «parte» da cui provengono con gli interessi generali della collettività. I candidati così selezionati contribuirebbero ad assicurare la formazione di maggioranze parlamentari e di governo che, in virtù dell'assenza di condizionamenti settoriali provenienti dai rappresentati, assicurerebbero la rappresentanza nella sua accezione più alta, cioè come responsabilità indipendente (Sartori 1987a).

In realtà è raro che $i$ partiti reclutino $i$ candidati da collocare nelle proprie liste controllando prioritariamente le loro attitudini a farsi interpreti degli interessi generali. Semmai, questo è un requisito distintivo che qualifica i leaders politici nei partiti e nelle assemblee elettive, mentre gli altri potranno imparare dopo la loro eventuale elezione operando all'interno delle istituzioni con esiti diversi commisurati alle inclinazioni. I partiti, invece, selezionano i candidati secondo criteri meno universalistici e più condizionati dall'obiettivo contingente di catturare il voto dell'elettore. Nelle pagine che seguono assumeremo che i partiti abbiano adottato tre principali criteri di selezione; ne illustreremo rapidamente le caratteristiche e controlleremo poi, attraverso l'esame dei candidati, in che misura e con quali scopi siano stati adottati dalle tre coalizioni.

Fatta salva l'esigenza di ottenere l'adesione ai programmi del partito e, auspicabilmente, ma non necessariamente, alla sua ideologia, le élites partitiche reclutano i candidati utilizzando tre principali criteri: la selezione autoreferente; la rappresentatività; l'appello alle figure sociali più predisposte al reclutamen- 
to ${ }^{1}$. Il primo criterio induce i partiti a tener conto delle aspettative che maturano al loro interno tra i funzionari, gli amministratori locali, gli uomini del sottogoverno, i parlamentari, i quali aspirano ad ottenere la (ri)candidatura e l'appoggio attivo durante la campagna elettorale come riconoscimento della loro fedeltà e a coronamento di una carriera di dedizione al partito. Il secondo criterio nasce dalla necessità di selezionare candidati con una forte rappresentatività sociologica, cioè che riflettano per il loro mestiere, o per gli interessi che rapppresentano, le caratteristiche sociali degli elettori a cui chiedono il voto. Quest'ultimi sono, infatti, più facilmente indotti a identificarsi con chi gli è simile per provenienza e professione e perciò a votare per i partiti nelle cui liste risultano candidati. Il terzo criterio è l'appello alle figure sociali più facilmente ed utilmente disponibili a fornire candidati ai partiti. Alcune figure sociali posseggono, infatti, competenze, tempo, prestigio, facilità di contatti sociali in misura tale da predisporle meglio di altre ad accettare l'invito dei partiti e, soprattutto, a competere con chances di successo nel confronto elettorale. A tali figure i partiti sono, perciò, in qualche misura obbligati a ricorrere per costruire liste elettorali competitive.

Ciascuno di questi criteri di selezione delle candidature comporta vantaggi e rischi. La scelta di selezionare candidati provenienti soprattutto dall'interno del partito può favorire il rafforzamento dell'organizzazione perché incentiva i militanti ad impegnarsi per poi ottenere la ricompensa della candidatura. Il rischio è che si creino delle oligarchie di professionisti della politica che acquisiscano il controllo dei canali di accesso alle cariche pubbliche riducendo, di conseguenza, le possibilità di quella «circolazione delle élites» che è un requisito indispensabile per il mantenimento del buono stato di salute di una democrazia liberale. Non è, però, scontato che le leadership partitiche, che hanno il controllo dei canali di selezione, siano del tutto consapevoli dei pericoli connessi alla riproduzione di un

${ }^{1}$ Naturalmente i partiti devono spesso combinare con questi anche altri e più pragmatici criteri di selezione dei candidati che rispondono alla necessità di trovare soluzioni a problemi delicati quale, per esempio, la preparazione di un cartello elettorale che ospiti al suo interno gli esponenti di tutte le liste che ne fanno parte senza creare risentimenti o malumori in nessuno dei partecipanti alla coalizione. Come è noto tutti $i$ partiti prima dell'avvio della campagna elettorale di marzo hanno dovuto misurarsi con questo tipo di problema e trovare soluzioni che armonizzassero le esigenze delle diverse liste. 
sistema di rappresentanza autoreferente. In ogni caso possono anche correre il rischio di irrigidire il sistema di ricambio del ceto politico parlamentare fino a quando riescono ad ottenere sufficienti consensi elettorali per sé e per i loro partiti. In periodi di transizione politica, come è quella che ha preceduto ed accompagnato la campagna elettorale, le élites partitiche devono, però, prendere atto della necessità di favorire l'accesso nelle assemblee elettive nazionali a nuove figure sociali. In questi casi, $i$ partiti devono riconsiderare il problema del peso che i politici di professione possono avere nelle liste elettorali, e trovare soluzioni che concilino l'esigenza di premiare i fedeli con quella di aprire la porta anche ad altri.

Il criterio di selezionare i candidati sulla base del principio della rappresentatività offre alle élites partitiche sia il vantaggio di una più facile identificazione tra elettore e candidato che l'opportunità di lanciare un messaggio esplicito a tutti coloro che per condizioni oggettive connesse alle loro attività, e/o per la condivisione soggettiva delle aspettative di quella classe, possono riconoscersi nel programma presentato da candidati simili a loro. Gli svantaggi che derivano dal seguire questa strada sono presto detti: il partito rischia di farsi identificare come rappresentante di uno specifico segmento sociale e quindi di apparire incapace di rappresentare i tanti e diversificati interessi di una società di massa. La presentazione di candidati provenienti da una sola professione o classe sociale può portare a non gradite sconfitte elettorali, a meno che non si coltivi la limitata ambizione di occupare uno spazio politico minoritario all'interno del sistema partitico.

Infine, la strada di privilegiare candidati provenienti dalle figure sociali predisposte, se venisse perseguita come unico criterio per comporre le liste, comporterebbe il rischio di presentare candidati provenienti prevalentemente da élites sociali o da figure con profili professionali molto particolari, che per ciò stesso incontrerebbero difficoltà ad ottenere un ampio consenso tra gli elettori.

Da quanto si è detto appare evidente che nessun partito sarà disposto a privilegiare esclusivamente l'uno o l'altro dei tre criteri che possono guidare le scelte delle élites partitiche nella selezione dei candidati. Prevarranno, invece, soluzioni che sono il frutto del contemperamento dei tre criteri appena enucleati secondo combinazioni differenti condizionate dalla collocazione politica e dalle tradizioni organizzative dei diversi partiti. 


\section{Le professioni dei candidati}

Uno sguardo d'insieme alle attività professionali dichiarate dai candidati nei collegi uninominali nelle elezioni del marzo 1994 consente di individuare diversi elementi di similarità con le analoghe attività dichiarate dai parlamentari italiani nelle legislature precedenti, e riscontrabili anche nelle occupazioni attribuibili al ceto parlamentare delle maggiori democrazie europee $^{2}$. Accanto a queste similarità si notano anche alcune significative novità che consentono di individuare una importante svolta nella composizione dei candidati che ha avuto sicuramente delle ripercussioni nella composizione sociologica del ceto politico parlamentare della XII legislatura.

Naturalmente non è metodologicamente ortodosso il confronto tra candidati e parlamentari per ricostruire le peculiarità dei profili sociologici dei primi. D'altra parte questa è una scelta obbligata in mancanza di ricerche sui candidati in consultazioni elettorali precedenti a quella da poco conclusa.

Gli elementi di similarità con il passato sono dati dalla presenza delle consuete figure sociali che ormai da diversi decenni popolano con la loro presenza le assemblee elettive delle liberal-democrazie. Innanzitutto vanno considerati i liberi professionisti. Costoro (avvocati, medici, altri professionisti) costituiscono da soli oltre il $30 \%$ di tutti i candidati per le elezioni del XII parlamento (Tab. 4). L'incidenza percentuale di questo gruppo è abbastanza vicina a quella riscontrata, dalle poche ricerche compiute sull'argomento, tra i parlamentari liberi professionisti attivi lungo il corso dell'esperienza dell'Italia repubblicana (Di Palma e Cotta 1986, 53; Mastropaolo 1993, 90). Tradizionalmente, $\mathrm{i}$ liberi professionisti hanno fornito le figure professionali maggiormente predisposte a farsi reclutare nelle liste elettorali. In passato gli avvocati costituivano il gruppo più numeroso, ma hanno continuato a diminuire sia tra le file dei candidati che degli eletti. Nel nostro campione gli avvocati sono alla pari con i medici i quali, sebbene anch'essi in passato sempre presenti tra i candidati e gli eletti, non sono mai stati così numerosi nelle liste come nelle ultime elezioni.

Oltre ai liberi professionisti tra le figure sociali predisposte

2 Sul ceto parlamentare italiano oltre al noto lavoro di Cotta (1979) si può menzionare anche il recente studio di Mastropaolo (1993). 
vanno considerati i docenti universitari e gli insegnanti. Le risorse ed opportunità che rendono i primi più disponibili di altre figure sociali a farsi reclutare nelle liste elettorali sono sostanzialmente analoghe a quelle di cui possono avvalersi i liberi professionisti (competenze, tempo, prestigio, facilità di contatti sociali). Gli insegnanti posseggono, invece, solo in parte tali risorse e devono frequentemente misurarsi con problemi di reddito e di scarsa gratificazione professionale. Questi fattori possono indurre gli insegnanti all'attivismo politico per rilanciare le loro chances di mobilità sociale e di realizzazione esistenziale che la professione di origine non sempre è in grado di offrire (Suleiman 1986, 98). Va, infine, sottolineato che gli insegnanti nel nostro campione sono i candidati in assoluto più numerosi. Il dato testimonia della crescente presenza di questa categoria professionale nelle liste elettorali ed in parlamento ed è da correlare, come vedremo meglio più avanti, con lo sviluppo dei partiti di massa e con il declino relativo della presenza delle professioni liberali.

Un discorso a parte va fatto per i candidati magistrati che per la particolarità dei compiti istituzionali e professionali loro affidati non possono di solito disporre delle opportunità dei liberi professionisti. Infatti, nella esperienza dei parlamenti dell'Italia repubblicana il numero dei magistrati parlamentari è quasi irrilevante. La loro presenza nelle file dei candidati nelle elezioni di marzo si deve, perciò, probabilmente attribuire alla crescente politicizzazione che hanno di recente subito alcuni settori della magistratura.

$\mathrm{Nel}$ complesso, tra le figure professionali che scendono e le altre che salgono nel listino delle candidature elettorali, constatiamo una robusta presenza tra i candidati dei liberi professionisti e dei docenti di scuola media ed universitari, che indica come l'appello alle professioni predisposte sia stato adottato in misura notevole anche nelle ultime elezioni (Tab. 4).

Un altro gruppo di candidati che presenta una certa, relativa, omogeneità, è quello degli imprenditori e degli addetti al settore del cosiddetto lavoro autonomo. Ciò che li accomuna è il carattere privato delle loro attività professionali, il fatto che siano basate su iniziative di rischio, una comune mentalità caratterizzata dall'insofferenza verso le lentezze del settore pubbli$\mathrm{co}^{3}$. Per le caratteristiche del loro lavoro queste figure sono di

${ }^{3}$ L'attribuzione di un comune atteggiamento culturale ci ha consentito di colloca- 
TAB. 4. Le attività professionali dei candidati

\begin{tabular}{lrc}
\hline ATTIVITÀ & N. & $\%$ \\
\hline Avvocati & 126 & 11,5 \\
Medici & 125 & 11,4 \\
Altri professionisti & 88 & 8 \\
Insegnanti & 132 & 12 \\
Docenti universitari & 78 & 7,1 \\
Magistrati & 22 & 2 \\
Imprenditori & 79 & 7,2 \\
Dirigenti d'azienda & 38 & 3,5 \\
Commercianti e artigiani & 37 & 3,4 \\
Terziario privato* & 81 & 7,4 \\
Politici di professione & 88 & 8 \\
Giornalisti & 50 & 4,6 \\
Dipendenti pubblici & 65 & 5,9 \\
Sindacalisti & 26 & 2,4 \\
Pensionati & 31 & 2,8 \\
Altre attività & 30 & 2,8 \\
Totale & & 100 \\
\hline
\end{tabular}

* Comprende sia le attività autonome sia i dipendenti.

solito scarsamente inclini ad intraprendere l'attività politica che li distoglierebbe da un impegno vissuto in modo assorbente. Naturalmente le difficoltà e le disponibilità cambiano in relazione al tipo e alla dimensione dell'attività intrapresa. Gli ostacoli che incontra un piccolo operatore per dedicarsi all'impegno politico sono molto maggiori di quelli affrontati dal grande industriale con un'azienda finanziariamente solida ed affidata alla guida di un esperto management. Tuttavia, è anche nota la tradizionale ritrosia dei grandi industriali ad impegnarsi direttamente in politica. Ciò spiega perché la loro presenza non sia mai stata superiore al $2,5 \%$ del totale di tutti i parlamentari presenti nelle assemblee legislative dell'Italia repubblicana. Percentuali anche più modeste sono attribuibili agli addetti nel lavoro autonomo. Dati tali precedenti, va considerata come un

re nel terziario avanzato insieme ai titolari di attività indipendenti (es.: l'agente immobiliare e il proprietario di un'agenzia assicurativa) e alle «nuove» professioni (l'informatico, l'esperto di marketing, il conduttore televisivo, l'interprete), anche i dirigenti e gli impiegati di aziende di piccole dimensioni. Come si vedrà meglio più avanti la collocazione di questi ultimi in un medesimo raggruppamento che comprende anche gli industriali apparirà giustificata dalle analoghe collocazioni politiche della maggioranza di queste figure professionali. 
fatto di particolare novità la numerosa presenza di questo tipo di candidati. Ben il $21,5 \%$ dei candidati del nostro campione svolge attività imprenditoriali o nel lavoro autonomo (Tab. 4). Il fatto che, a differenza del passato, una così cospicua rappresentanza dei ceti medi produttivi abbia deciso di partecipare direttamente alla competizione elettorale è spiegabile (come vedremo tra poco) con il tentativo compiuto da alcune formazioni politiche di utilizzare come criterio principale di reclutamento dei candidati quello della rappresentatività.

Dopo le libere professioni, gli insegnanti e il lavoro autonomo, il quarto gruppo di candidati che si contraddistingue per rilevanza numerica $(10 \%)$ è quello costituito dai politici di professione, nei quali comprendiamo anche i sindacalisti (Tab. 4). Definiamo politici di professione quelli che, prima di candidarsi alle elezioni, hanno fatto dell'attività politica il loro mestiere, impegnando tutto il loro tempo lavorativo, o la maggior parte di esso, nella politica. L'attività professionale di questo tipo di candidato si qualifica dunque per l'assenza di un'occupazione extra politica. Tali candidati sono presenti in misura consistente nelle liste elettorali se i partiti decidono di far prevalere il criterio della selezione autoreferente per consentire il completamento di un cursus honorum il cui approdo più elevato è il Parlamento, o per neutralizzare le difficoltà politiche che derivano dalla scarsa capacità del partito stesso di acquisire alle sue liste candidati prestigiosi o rappresentativi.

Nel nostro campione il numero dei politici di professione, almeno per il momento, non pare particolarmente rilevante (Tab. 4). Ciò potrebbe far ritenere che i partiti non hanno adottato il criterio della selezione autoreferente. Ma bisogna diffidare di questa prima impressione perché, come il lettore esperto sa, il politico di professione può anche svolgere un'attività professionale che è, o è divenuta, del tutto strumentale allo svolgimento dell'attività politica. A tale occupazione i politici di professione italiani «hanno avuto accesso magari attraverso un avallo politico, l'hanno funzionalizzata alle loro ambizioni e di rado contemplano la possibilità di svolgerla davvero» (Mastropaolo 1993, 104). Quali sono concretamente le attività professionali strumentali vedremo meglio più avanti, quando cercheremo di quantificare l'effettiva consistenza della presenza dei politici di professione tra i nostri candidati. Per il momento conviene soffermarsi ad individuare quale combinazione dei tre criteri di reclutamento che abbiamo illustrato sia stata adottata da ciascuna coalizione e dalle maggiori liste ad esse collegate. 
La selezione

Da un primo sguardo alla tabella 5 appare evidente che le tre coalizioni hanno attivamente praticato l'appello alle professioni predisposte, in particolare ai liberi professionisti. L'elevata presenza dei liberi professionisti tra i candidati del nostro campione (il $31 \%$ del totale), che abbiamo già constatato, lasciava prevedere che l'appello fosse stato rivolto soprattutto a loro. Bisogna, d'altra parte, rilevare che tale scelta è stata perseguita con maggiore determinazione dal $\mathrm{Pdl}-\mathrm{Pbg}$, all'interno del quale i liberi professionisti costituiscono oltre il $36 \%$ del totale dei candidati, e dal Patto $(33,1 \%)$, mentre nella coalizione di sinistra l'incidenza di queste figure professionali scende al $20,7 \%$ (Tab. 5).

In realtà la sinistra ha abbinato all'appello alle professioni predisposte una maggiore attenzione al mondo della scuola (docenti universitari e insegnanti) da cui proviene il numero più elevato di candidati con professioni sociologicamente omogenee rilevabile nella coalizione progressista (il 28,7\%) (Tab. 5). In

ТАВ. 5. Le attività professionali dei candidati, distinti per coalizione (\%)

\begin{tabular}{lcccc}
\hline ATTIVITÀ & \multicolumn{3}{c}{ COALIZIONI } & \\
\cline { 2 - 4 } & Pgr & PpI & Pdl-Pbg & Tot. \\
Avvocati & 4,5 & 9,3 & 17,1 & 11,5 \\
Medici & 9,7 & 13,8 & 11,1 & 11,4 \\
Altri professionisti & 6,5 & 10 & 7,9 & 8 \\
Insegnanti & 14,5 & 17,2 & 7,5 & 12 \\
Docenti universitari & 14,2 & 7,3 & 2,6 & 7,2 \\
Magistrati & 4,5 & 1,7 & 0,6 & 2 \\
Imprenditori & 2,3 & 5,9 & 11,2 & 7,2 \\
Dirigenti d'azienda & 1 & 5,2 & 4 & 3,5 \\
Commercianti e artigiani & 1 & 2,2 & 5,6 & 3,3 \\
Terziario privato* & 3,2 & 5,2 & 11,3 & 7,4 \\
Politici di professione & 17,7 & 3,4 & 4,6 & 8 \\
Giornalisti & 4,8 & 4,8 & 4,3 & 4,6 \\
Dipendenti pubblici & 5,2 & 9,3 & 4,4 & 6 \\
Sindacalisti & 5,5 & 1,7 & 0,8 & 2,4 \\
Pensionati & 2,5 & 1,7 & 3,6 & 2,8 \\
Altre attività & 2,9 & 1,3 & 3,4 & 2,7 \\
Totale & & & & 100 \\
(N) & 100 & 100 & 100 & $(1.096)$ \\
\hline
\end{tabular}

* Si veda la tabella 4. 
modo in parte analogo si è comportato il Patto, i cui candidati per il $24,5 \%$ provengono anch'essi dal settore dell'istruzione (con gli insegnanti che da soli sono più del $17 \%$ del totale dei candidati). Decisamente più modesta è, invece, l'incidenza dei candidati provenienti dalla scuola nel Pdl-Pbg (il 10\%).

Questi primi dati sembrerebbero confermare una tendenza di lungo periodo, rilevata già da tempo (Sartori 1987b; Di Palma e Cotta 1986) nel rapporto tra partiti e classi medie: gli strati superiori entrano di preferenza nei ranghi del ceto politico dei partiti di destra mentre gli strati inferiori delle classi medie trovano un'opportunità di mobilità sociale inserendosi nelle strutture dei partiti di massa dalle quali vengono successivamente proiettati nelle assemblee legislative. Tale tendenza sembrerebbe ulteriormente confermata nel nostro campione dalla rilevante presenza dei dipendenti pubblici tra $\mathrm{i}$ candidati del Patto $(9,3 \%)$ e, in misura più contenuta $(5,2 \%)$, tra quelli della coalizione di sinistra (Tab. 5). Se accettiamo questa interpretazione diviene, però, evidente che l'elevato numero di candidati provenienti dagli strati inferiori delle classi medie nelle coalizioni di centro e di sinistra non è più spiegabile esclusivamente alla luce del criterio dell'appello alle professioni predisposte. È, infatti, plausibile ritenere che gli strati inferiori delle classi medie si trovino in numero elevato tra $\mathrm{i}$ candidati delle coalizioni di centro e di sinistra non solo perché sono più pronti di altri ceti sociali ad attivarsi politicamente, ma anche perché sono quelli più adatti ad alimentare la formazione e la riproduzione del ceto dei politici di professione. In altri termini, in questo caso i partiti avrebbero sovrapposto al criterio dell'appello alle professioni predisposte quello della selezione autoreferente che avrebbe finito per prevalere sul primo.

Il problema è abbastanza complesso e può essere chiarito solo disponendo di ulteriori informazioni sui candidati che verranno fornite più avanti. Per ora conviene spostare l'attenzione sulle altre figure sociali a cui i partiti si sono rivolti per il reclutamento, in particolare i candidati collegabili alle attività imprenditoriali e al lavoro autonomo. Costoro si sono distribuiti nelle tre coalizioni secondo differenze che ricalcano le presenze dei candidati provenienti dalle libere professioni, cioè meno a sinistra e più a destra. Tuttavia, in questo caso la differenza nelle proporzioni è molto più sensibile: ad un candidato di sinistra proveniente da queste figure sociali ne corrispondono più di quattro nella coalizione di destra $(32,1 \%$ contro il $7,5 \%)$ (Tab. 
5). Il Patto ha un rapporto meno sfavorevole, ma l'incidenza dei suoi candidati su queste attività $(18,5 \%)$ rimane, comunque, ben lontana da quella dei candidati della destra.

Quale criterio è stato adottato da quest'ultima per accogliere tra le sue file un numero così elevato di candidati provenienti da attività che per le loro caratteristiche rendono, come si è detto, difficile l'impegno in politica? L'ipotesi più plausibile, e verosimile, è che la coalizione di destra abbia adottato il criterio della rappresentatività, cioè abbia cercato di valorizzare la presenza di candidati provenienti dall'imprenditoria e dal lavoro autonomo. In altri termini, le formazioni della destra hanno tradotto in pratica le tante dichiarazioni dei mesi precedenti la campagna elettorale che sottolineavano la necessità di incoraggiare $\mathrm{i}$ ceti medi produttivi ad entrare direttamente nell'arena politica per sostituirsi ad una partitocrazia delegittimata.

L'altra area di attività professionale alla quale i partiti si sono rivolti per reclutare $\mathrm{i}$ loro candidati è quella dei politici di professione. Il criterio di reclutamento che viene adottato per queste figure sociali è, come si è già anticipato, la selezione autoreferente che, nel nostro caso, è stata perseguita soprattutto dalla coalizione di sinistra con il risultato di collocare nelle sue liste (considerando anche $\mathrm{i}$ sindacalisti) il 23,2\% di candidati politici di professione. Decisamente più modesta appare, secondo i dati della tabella 5 , la quota di politici di professione attribuibile al Pdl-Pbg e al Patto.

$\grave{E}$ ora opportuno controllare in quale misura i maggiori partiti hanno selezionato i candidati secondo la combinazione di criteri preferita dalla coalizione di appartenenza. Ciò ci consentirà di controllare meglio, dall'interno, le strategie di reclutamento adottate da ciascuna coalizione. Per le formazioni costituenti il Patto per l'Italia non abbiamo informazioni differenziate e perciò rimandiamo a quanto appena rilevato sulla coalizione di centro. Riguardo alla coalizione di sinistra lirnitiamo, invece, la nostra analisi alle scelte effettuate dal Pds ma avremo modo di menzionare indirettamente quelle compiute anche dalle formazioni minori; per il $\mathrm{Pdl}-\mathrm{Pbg}$ esamineremo le tre maggiori formazioni.

Cominciando dal Pds è facile constatare che la composizione socio-professionale dei suoi candidati ha le stesse caratteristiche riscontrate nella coalizione di sinistra, ma tutte accentuate. I candidati del $\mathrm{Pds}$ sono, infatti, prevalentemente, ed in eguale misura $(31,7 \%)$, sia insegnanti e docenti universitari che politici 
TAB. 6. Le attività professionali dei candidati, distinti per liste (\%)

\begin{tabular}{lcrrr}
\hline ATTIVITA & \multicolumn{3}{c}{ LISTE } \\
\cline { 2 - 5 } & Pds & Ln & Fi & An \\
Avvocati & 2,4 & 11,6 & 11,8 & 21,6 \\
Medici & 7,3 & 8,9 & 16,4 & 8,8 \\
Altri professionisti & 4,1 & 12,5 & 5,5 & 4,4 \\
Insegnanti & 15,4 & 9,8 & 5,5 & 7,5 \\
Docenti univ. & 16,3 & 4,5 & 4,5 & 1,3 \\
Magistrati & 4,9 & & 1,7 & 0,4 \\
Imprenditori & & 8,9 & 20,9 & 8,4 \\
Dirigenti d'azienda & 0,8 & 5,4 & 6,4 & 2,2 \\
Commerc. e artigiani & 0,8 & 8 & 2,7 & 4,8 \\
Terziario privato* & 3,3 & 16,2 & 7,3 & 12,7 \\
Politici di profess. & 29,3 & 5,4 & 3,5 & 4,8 \\
Giornalisti & 3,3 & 2,7 & 4,5 & 4,8 \\
Dipendenti pubblici & 4 & 2,7 & 4,5 & 5,3 \\
Sindacalisti & 2,4 & & & 1,8 \\
Pensionati & 3,3 & 1,8 & 0,9 & 6,2 \\
Altre attività & 2,4 & 1,8 & 0,9 & 4 \\
Totale & 100 & 100 & 100 & 100 \\
(N) & $(123)$ & $(112)$ & $(110)$ & $(227)$ \\
\hline
\end{tabular}

* Si veda la tabella 4.

di professione, mentre i liberi professionisti e gli addetti all'imprenditoria e al lavoro autonomo sono presenti in misura anche minore di quella riscontrata a livello di coalizione (Tab. 6). Ciò significa che $\mathrm{i}$ candidati provenienti da tali attività sono stati reclutati prevalentemente dalle formazioni minori della coalizione progressista. Da notare che nessun imprenditore risulta collegato alle liste del Pds, perché lo è con quelle di Alleanza democratica.

Riguardo al Pdl-Pbg i candidati liberi professionisti risultano tutti al di sopra del $33 \%$ nelle tre maggiori liste alleate dello schieramento di destra ${ }^{4}$ con una loro più accentuata presenza in An, dovuta all'elevato numero di avvocati candidati in quella formazione (Tab. 6). Gli avvocati sono presenti in misura rilevante anche nella $\mathrm{Ln}$ e in Fi ma la prima ha più candidati tra gli

${ }^{4}$ La percentuale relativa a tutta la coalizione, che è superiore al $36 \%$ (Tab. 5), si spiega con il fatto che i candidati del Centro cristiano democratico, non considerati nella tabella 6 ma presenti nel nostro campione, provengono per quasi il $50 \%$ dalle professioni liberali. Ciò contribuisce ad elevare l'incidenza percentuale dei liberi professionisti a livello di coalizione in misura superiore a quella registrata in $\mathrm{Fi}$ e $\mathrm{Ln}$. 
«altri» professionisti e Fi tra i medici. Nel secondo sottogruppo determinante per la formazione delle liste del $\mathrm{Pdl}-\mathrm{Pbg}$, cioè quello del lavoro autonomo e imprenditoriale, abbiamo una significativa conferma di quanto era prevedibile aspettarsi: la maggioranza dei candidati provenienti dal lavoro autonomo è collegato alle liste della $\mathrm{Ln}$ e di $\mathrm{Fi}$, con una percentuale che nella prima è del $38,5 \%$ e nella seconda di poco inferiore. Decisamente più contenuta è la presenza di questo tipo di candidati nelle liste di An (quasi 10 punti in meno rispetto alla $\mathrm{Ln}$ ) ma comunque sensibilmente superiore rispetto a quella riscontrata nel Patto e nel Pds (Tab. 6). Vale, inoltre, la pena di sottolineare che, sempre nell'ambito di questo sottogruppo, i candidati imprenditori sono più numerosi in Fi mentre nella $\mathrm{Ln}$ e in An lo sono gli addetti al terziario privato. Scarsa è la presenza nel Pdl-Pbg dei politici di professione, specie per $\mathrm{Fi}(3,5 \%)$ che è formazione di nuova costituzione. Evidentemente, in questo caso i pochi politici di professione provengono da altre formazioni già preesistenti. Più numerosi sono, invece, i candidati politici nella Lega, nella quale si sta formando un ceto politico locale e nazionale.

Ricapitoliamo riportando l'analisi a livello di coalizione. Il $70 \%$ dei candidati della coalizione di destra è costituito da liberi professionisti e da addetti all'imprenditoria diffusa e al lavoro autonomo (Tab. 5). La sinistra ha, invece, i suoi punti di forza nel pubblico impiego, dove recluta candidati in misura maggiore tra le fasce alte e inferiori dei ceti medi che lavorano nel mondo della scuola, e tra i politici di professione. Il Patto si distingue dalle altre due coalizioni perché assomiglia un po' all'una e all'altra. Infatti, la sua maggior fonte di reclutamento proviene dalle libere professioni, alla stessa stregua del Pdl-Pbg, ma si differenzia da questo perché il suo secondo, più importante, serbatoio sociale di candidati non è il lavoro autonomo bensì - come per la sinistra - il mondo della scuola; bassa è, infine, la quota di candidati del Patto classificabili come politici di professione. In sintesi, i candidati del Patto si distribuiscono in modo meno squilibrato di quelli della sinistra e del $\mathrm{Pdl}-\mathrm{Pbg}$ sui tre grandi sottogruppi di attività professionali che abbiamo evidenziato nelle tabelle 4 e 5 quasi a confermare l'ambizione di questa coalizione a collocarsi al centro del nuovo sistema partitico. 


\section{Quanti politici di professione?}

L'analisi fin qui svolta ha offerto numerosi elementi di conoscenza sulle caratteristiche sociologiche dei candidati, sulla loro presenza nelle tre coalizioni, sulle strategie di reclutamento adottate da ciascuna coalizione e dalle liste collegate. Per quest'ultimo aspetto sono, tuttavia, emersi alcuni problemi di interpretazione relativi all'effettiva rilevanza avuta da certi criteri di reclutamento rispetto ad altri. Sembrerebbe, infatti, dai dati finora esaminati, che la selezione autoreferente dei candidati sia stata praticata prevalentemente all'interno della coalizione progressista e dal Pds in particolare. In realtà questo tipo di conclusione, ancorché verosimile, appare tuttavia insufficiente perché finisce per sottovalutare la dimensione di un fenomeno, quello dei candidati politici di professione, che ha una consistenza maggiore. Dovremo, perciò, ora, compiere un ulteriore passo avanti ed introdurre altre informazioni sui candidati che consentano di controllare: a) se la selezione autoreferente sia stata praticata, oltre che dal Pds e dalla coalizione di sinistra, anche dalle altre formazioni in misura maggiore a quella finora accertata; e $b$ ) evidenziare le diverse figure di politico di professione che risultano mimetizzate dietro l'esercizio di altre attività.

Per raggiungere questi obiettivi abbiamo cercato innanzitutto di individuare tutti $i$ candidati che nel nostro campione hanno ricoperto cariche elettive a livello locale o sono stati parlamentari (Tab. 7). Costoro, complessivamente 557 candidati, sono presenti in misura significativa in tutte e tre le coalizioni. Le cariche più numerose sono quelle di consigliere comunale, parlamentare e sindaco. La loro somma rende ragione di oltre il $73 \%$ delle cariche ricoperte.

A livello di coalizioni si nota una sensibile differenza nel numero di cariche parlamentari attribuibili ai candidati collegati alle diverse liste. Tra i progressisti le cariche parlamentari ammontano a quasi il $40 \%$ di tutte le cariche elettive dei candidati della coalizione di sinistra. Decisamente più ridotta, rispetto a quella della coalizione progressista, è la quota di cariche parlamentari $(24 \%)$ attribuibile ai candidati del Patto (Tab. 7). Questo dato confermerebbe le dichiarazioni e le previsioni della vigilia elettorale, con il Patto e il Patto Segni impegnati in un laborioso negoziato per «ripulire» da parlamentari discussi le liste collegate alla nascente coalizione. 
TAB. 7. Cariche elettive ricoperte dai candidati prima elo al momento della candidatura distribuite per coalizione, ed in Alleanza nazionale. Valori assoluti

\begin{tabular}{|c|c|c|c|c|c|}
\hline & $\mathrm{Pgr}$ & PpI & Pdl-Pbg & (An) & Tot. \\
\hline $\begin{array}{l}\text { Parlamentare } \\
\text { Pres. Regione } \\
\text { Pres. Provincia } \\
\text { Sindaco } \\
\text { Assessore regionale } \\
\text { Assessore provinciale } \\
\text { Assessore comunale } \\
\text { Consigliere regionale } \\
\text { Consigliere provinciale } \\
\text { Consigliere comunale } \\
\text { Altre cariche }\end{array}$ & $\begin{array}{r}101 \\
2 \\
4 \\
35 \\
5 \\
7 \\
24 \\
10 \\
7 \\
60 \\
3\end{array}$ & $\begin{array}{r}54 \\
3 \\
3 \\
43 \\
7 \\
6 \\
25 \\
8 \\
9 \\
45 \\
5\end{array}$ & $\begin{array}{r}16 \\
2 \\
5 \\
16 \\
15 \\
15 \\
110 \\
6\end{array}$ & $\begin{array}{r}(4) \\
(1) \\
(2) \\
(3) \\
(11) \\
(11) \\
(88) \\
(3)\end{array}$ & $\begin{array}{r}219 \\
5 \\
7 \\
91 \\
14 \\
18 \\
65 \\
33 \\
31 \\
215 \\
14\end{array}$ \\
\hline $\begin{array}{l}\text { Totale cariche } \\
\text { (n. candidati) }\end{array}$ & $\begin{array}{c}258 \\
(193)\end{array}$ & $\begin{array}{c}208 \\
(158)\end{array}$ & 249 & (154) & $\begin{array}{c}715 \\
(557) \\
\end{array}$ \\
\hline
\end{tabular}

Ancora più ridotta è la quota $(20 \%)$ di cariche parlamentari sul totale delle cariche attribuite ai candidati del Pdl-Pbg (Tab. 7), presumibilmente a causa delle scarse precedenti esperienze politiche dei candidati di Fi (che più avanti apparirà ancora più evidente) e per la necessità della Ln di sacrificare qualcuno dei suoi parlamentari per far posto ai candidati della lista alleata nelle regioni del nord del paese. Ciò spiega anche perché An, nell'ambito del Pdl-Pbg, abbia finito per schierare un così elevato numero di parlamentari: «costretta» a correre da sola in tutte le regioni del centro-nord, e in competizione con Fi in diversi collegi della Campania, ha avuto l'opportunità di candidare, come si è detto, tutti i suoi parlamentari (con l'eccezione di due) che, non avendo mai ricoperto cariche di governo o incarichi pubblici di rilievo, risultavano al riparo dal pericolo di incorrere in disavventure giudiziarie. Rispetto alle altre due liste della destra, An ha anche avuto il vantaggio di essere stata l'unica formazione nel $\mathrm{Pdl}-\mathrm{Pbg}$ a poter disporre di una struttura organizzativa di base estesa su tutto il territorio nazionale che era quella del Msi. Ciò spiega perché l'80\% delle cariche di consigliere comunale del $\mathrm{Pdl}-\mathrm{Pbg}$ risultano ricoperte da candidati di An (Tab. 7).

Le cariche di consigliere comunale sono state ricoperte in modo significativo anche dai candidati delle altre due coalizioni, ma in misura minore che nel $\mathrm{Pdl}-\mathrm{Pbg}$. Ciò perché nel Pds e nel Patto i consiglieri comunali hanno dovuto spesso far posto 
a sindaci ed assessori, che erano meno numerosi nei ranghi del Msi a causa dello scarso potenziale di coalizione per la formazione delle giunte locali che questo partito ha avuto in passato. Anche i candidati di Fi hanno potuto vantare a proprio credito poche cariche amministrative, mentre la Lega non poteva sguarnire dei suoi uomini migliori i comuni e i centri di potere locale la cui buona conduzione è un obiettivo strategico per la sopravvivenza stessa di questa formazione.

Nel complesso, il numero delle cariche politiche attribuibile ai candidati delle tre coalizioni indica che a livello delle cariche parlamentari (pari al 18,5\% del totale) (Tab. 8) i progressisti e, nel Pdl-Pbg, An hanno cambiato molto meno che il Patto, il quale ha sostituito i parlamentari meno presentabili con i sindaci provenienti dalla robusta rete del potere locale democristiano. Un altro elemento di notevole rilievo, che vale la pena sottolineare, è l'incidenza complessiva delle cariche - locali e parlamentari - attribuibili ai candidati di An (quasi il 64\%) sul totale delle cariche dei candidati del $\mathrm{Pdl}-\mathrm{Pbg}$, a conferma della consistenza della struttura organizzativa di questa formazione (Tab. 7).

Cosa ci dicono le cariche politiche che abbiamo attribuito ai nostri candidati? In realtà sarebbe azzardato considerare politici di professione tutti coloro che le hanno ricoperte perché $\mathrm{i}$ nostri dati non ci danno la durata degli incarichi. Perciò non siamo in grado di distinguere tra i neofiti, magari entrati in politica in occasione delle ultime elezioni amministrative, e quelli con più lunga esperienza alle spalle. Si può, tuttavia, ragionevolmente supporre che, almeno per quanto riguarda le cariche locali, è improbabile che i partiti abbiano candidato al parlamento i neo-eletti delle amministrative del novembre 1993. È,

TAB. 8. Candidati con cariche elettive e partitiche ricoperte prima elo al momento della candidatura

\begin{tabular}{lcc}
\hline Cariche & N & $\begin{array}{c}\text { Candidati } \\
\text { \% (su 1.204 candidati) }\end{array}$ \\
\hline Solo cariche parlamentari & 150 & 12,5 \\
Solo cariche elettive locali & 335 & 27,8 \\
Sia cariche elettive che parlamentari & 72 & 6 \\
Solo cariche partitiche & 44 & 3,6 \\
Totale & 601 & 49,9 \\
\hline
\end{tabular}


invece, più probabile che $\mathrm{i}$ candidati siano uomini con un certa esperienza amministrativa alle spalle, anche perché è ormai quasi scomparso il politico di professione che è esclusivamente un politico di apparato, cioè titolare solo di cariche partitiche: nel nostro campione sono solo $44 \mathrm{i}$ candidati che hanno ricoperto esclusivamente cariche partitiche (Tab. 8). Piuttosto, è diventato un fattore determinante, ai fini di una possibile carriera politica nazionale, l'aver ricoperto cariche elettive locali. Ciò perché l'aver prestato «servizio» a livello locale, più che un fatto di fedeltà ed affidabilità, viene considerato un requisito utile per acquisire potere e visibilità e quindi risorse proprie per misurarsi in una competizione elettorale più ardua come è quella per l'ingresso in parlamento'.

Dunque, sebbene sia improbabile che i 601 candidati (Tab. 8) che hanno avuto esperienze politiche precedenti siano tutti politici di professione, è anche vero che tra di loro ci sono gli uomini della rete organizzativa di base delle diverse formazioni politiche e i leaders nazionali. Perciò è più che probabile che il sottogruppo dei 601 , pari al 50\% del totale dei candidati del nostro campione, sia costituito in larghissima maggioranza da politici di professione. Del resto le stime che in questi anni sono state effettuate per stabilire l'incidenza reale del fenomeno dei politici di professione sulla composizione del ceto politico parlamentare lo hanno valutato su percentuali sostanzialmente identiche alle nostre. Infine, non bisogna dimenticare che in tutti i processi politici che comportano la destrutturazione e ristrutturazione dei sistemi della rappresentanza politica i nuovi attori che entrano nell'arena politica a sostituire i vecchi fanno sempre leva sulle precedenti risorse organizzative che possono loro offrire le strutture delle formazioni «parenti» che esse sostituiscono, o sulle preesistenti reti associative che ne sostengono la formazione. Ciò vale sia per i partiti che per i gruppi di interesse, che per gli stessi movimenti collettivi. Ed è quanto hanno fatto anche i partiti politici italiani nelle elezioni di marzo per selezionare un ceto politico parlamentare più presentabile di quello che lo aveva preceduto.

\footnotetext{
5 Su questo punto insiste Mastropaolo $(1993,105)$ quando discute i curricula del ceto parlamentare italiano.
} 


\section{E quali?}

Si tratta ora di chiarire se si possa stabilire una qualche distinzione all'interno di questo gruppo di candidati che si è rivelato più esteso di quanto non sembrasse in un primo momento. Procederemo utilizzando alcune delle definizioni correnti sui politici di professione riproposte recentemente da Mastropaolo che ci serviranno per formulare qualche ipotesi sulla presenza diversificata di questa categoria nel nostro campione.

Il riesame critico delle informazioni sulle «professioni ufficiali» o di paravento, dichiarate dai politici di professione, consente di ipotizzare tre distinte varianti di questo tipo di candidato: a) il politico di professione in senso proprio che non svolge alcun'altra attività se non quella dichiarata; $b$ ) il politico di professione che svolge un'attività professionale strumentale a quella politica; $c$ ) il politico di carriera, cioè il candidato che svolge un'attività professionale non strumentale a quella politica ma qualificata, ed intraprende la carriera politica come attività aggiuntiva a quella precedente. Per questo tipo di candidato la professione originaria è un'attività che può essere ancora esercitata, nonostante gli impegni politici; dalla quale egli può continuare a ricavare una parte rilevante del suo reddito e che offre un approdo nel caso egli volesse smettere di fare attività politica (Mastropaolo 1993, 106).

Nel sottogruppo del nostro campione costituito da candidati con precedenti esperienze politiche si possono collocare tra $i$ politici di professione in senso proprio, oltre a quanti lo dichiarano, anche i sindacalisti. Nella coalizione di sinistra queste due categorie totalizzano ben il $38 \%$ dei candidati politici di professione, una percentuale molto più elevata di quella riscontrabile, per le medesime categorie, nelle altre due coalizioni (Tab. 9).

Politici di professione che operano al riparo di un'attività professionale strumentale si possono trovare tra gli insegnanti, $\mathrm{i}$ dipendenti pubblici, i giornalisti e, in qualche caso, tra i pensionati. In particolare, l'attività di insegnante sembra adatta ad offrire un'occupazione strumentale ai politici di professione. Ciò per le caratteristiche socio-professionali di tale attività, sulle quali si è già detto in precedenza, che possono favorire l'attivismo politico. Tali motivi spiegherebbero perché i candidati insegnanti sono molto presenti nelle tre coalizioni, anche se in misura più accentuata nel Patto per l'Italia e tra i Progressisti. Sui dipendenti pubblici e, in parte, i giornalisti, aggiungiamo 
TAB. 9. I candidati con cariche elettive elo partitiche distinti per coalizione ed attività professionale (\%)

\begin{tabular}{lrrr}
\hline \multirow{2}{*}{ ATTIVITÀ } & \multicolumn{3}{c}{ COALIZIONI } \\
\cline { 2 - 4 } & Pgr & PpI & Pdl-Pbg \\
\hline Avvocati & 3 & 8 & 19 \\
Medici & 8 & 15 & 7 \\
Altri professionisti & 4 & 7 & 9 \\
Insegnanti & 15 & 19 & 8 \\
Docenti universitari & 14 & 4 & 2 \\
Magistrati & 3 & 1 & 7 \\
Imprenditori & 1 & 4 & 3 \\
Dirigenti d'azienda & 1 & 5 & 6 \\
Commerc. e artigiani & 2 & 5 & 11 \\
Terziario privato* & 29 & 7 & 11 \\
Politici di professione & 3 & 3 & 3 \\
Giornalisti & 5 & 13 & 5 \\
Dipendenti pubblici & 9 & 4 & 2 \\
Sindacalisti & 2 & 4 & 4 \\
Pensionati & 1 & 1 & 3 \\
Altre attività & & 100 & 100 \\
& 100 & $(142)$ & $(211)$ \\
Totale & $(192)$ & &
\end{tabular}

* Si veda la tabella 4

che possono rivelarsi attività strumentali alle quali il politico di professione accede, e nelle quali fa carriera, grazie ai suoi incarichi politici. Nel nostro campione i dipendenti pubblici, che presumibilmente sono politici di professione, non mancano in nessuna delle tre coalizioni, ma risultano particolarmente numerosi nel Patto (Tab. 9). Costoro sono, probabilmente, ex democristiani a suo tempo assunti per meriti politici negli enti locali e statali che ora tentano la carriera parlamentare. Da notare, ancora, in tutte e tre le coalizioni, la modesta presenza di giornalisti probabili professionisti della politica.

Infine, candidati probabilmente divenuti politici di carriera sono quanti provengono dalle professioni liberali, dall'insegnamento universitario, in rari casi dalla magistratura e, in misura minore, dalle attività imprenditoriali e dal lavoro autonomo. Tra i nostri candidati i politici di carriera presenti nella coalizione di sinistra sembrano prevalentemente docenti universitari e, in misura molto minore, medici. Nel Patto per l'Italia questo gruppo di professionisti politici è, invece, costituito soprattutto $\mathrm{da}$ medici ed avvocati $\mathrm{e}$, in misura più limitata, da imprenditori 
ed addetti al lavoro autonomo. Nel Pdl-Pbg gli avvocati probabili politici di professione prevalgono nettamente sulle altre professioni liberali, che, pure, sono molto ben rappresentate. $\mathrm{Da}$ notare, ancora nel $\mathrm{Pdl}-\mathrm{Pbg}$, la forte presenza di addetti al lavoro autonomo ed imprenditoriale che potrebbero essere politici di professione (Tab. 9).

Ricapitolando su queste figure di probabili professionisti della politica, constatiamo che $i$ politici di professione in senso proprio (politici e sindacalisti), prevalgono nella coalizione di sinistra (39\%); quelli che sono anche titolari di attività professionali strumentali alla politica nel Patto (insegnanti e dipendenti pubblici, pari al $32 \%$ ); i politici di carriera, sembrano più numerosi, invece, nelle file del Pdl-Pbg sia tra il liberi professionisti $(35 \%)$ che nell'area delle attività imprenditoriali e del lavoro autonomo (27\%) (Tab. 9).

In complesso, nella coalizione di sinistra il numero maggioritario di candidati politici di professione è costituito da politici in senso proprio e da docenti, di scuola media ed universitaria (67\% del totale); nel Patto da liberi professionisti, insegnanti, dipendenti pubblici e politici in senso proprio (69\%); nel Pdl-Pbg dai liberi professionisti e dai ceti medi produttivi (in tutto il $61 \%$ ). Da ciò si deduce che i candidati politici di professione dei Progressisti e del Patto sono stati selezionati in modo massiccio dal pubblico impiego mentre tale fenomeno è decisamente più contenuto nel $\mathrm{Pdl}-\mathrm{Pbg}$.

Le tendenze che abbiamo individuato nella tabella 9 si precisano se esaminate a livello di liste (Tab. 10). Nel Pds il numero dei politici di professione in senso proprio aumenta ancora di più, anche se diminuisce la quota costituita dai sindacalisti, in cui è evidentemente determinante il numero dei candidati collegati alle liste minori (v. oltre). Aumentano anche gli insegnanti, cioè i politici che esercitano anche un'attività strumentale e, di poco, i docenti universitari, cioè i politici di carriera. Diminuisce ulteriormente nel $\mathrm{Pds}$, il peso, già modesto se paragonato a quello delle altre due coalizioni, dei candidati provenienti dalle libere professioni.

Per quanto riguarda le formazioni aderenti al $\mathrm{Pdl}-\mathrm{Pbg}$, c'è da constatare in Fi il numero decisamente modesto di candidati con precedenti esperienze politiche, che provengono prevalentemente dai ranghi dei liberi professionisti. Nella Ln prevalgono i politici di carriera e tra di essi una quota rilevante è costituita da imprenditori, addetti al terziario privato, commercianti, arti- 
TAB. 10. I candidati con cariche elettive e partitiche distinti per liste ed attività professionale (\%)

\begin{tabular}{|c|c|c|c|c|}
\hline \multirow[t]{2}{*}{ ATTIVITÀ } & \multicolumn{4}{|c|}{ LISTE } \\
\hline & Pds & $\operatorname{Ln}$ & $\mathrm{Fi}$ & An \\
\hline Avvocati & 2 & 6 & 26 & 25 \\
\hline Medici & 6 & 8 & 5 & 6 \\
\hline Altri professionisti & 4 & 16 & 11 & 4 \\
\hline Insegnanti & 16 & 10 & & 9 \\
\hline Docenti univ. & 15 & 4 & 11 & 1 \\
\hline Magistrati & 3 & & & \\
\hline Imprenditori & & 12 & 11 & 5 \\
\hline Dirigenti d'azienda & & 2 & & 3 \\
\hline Commerc. e artigiani & & 8 & & 5 \\
\hline Terziario privato* & 3 & 12 & 5 & 14 \\
\hline Politici di profes. & 37 & 12 & 21 & 9 \\
\hline Giornalisti & 1 & 2 & & 3 \\
\hline Dipendenti pubblici & 3 & 2 & 11 & 5 \\
\hline Sindacalisti & 3 & & & 3 \\
\hline Pensionati & 4 & 4 & & 4 \\
\hline Altre attività & 2 & 4 & & 4 \\
\hline $\begin{array}{l}\text { Totale } \\
\text { (N) }\end{array}$ & $\begin{array}{l}100 \\
(98)\end{array}$ & $\begin{array}{l}100 \\
(51)\end{array}$ & $\begin{array}{l}100 \\
(19)\end{array}$ & $\begin{array}{c}100 \\
(120)\end{array}$ \\
\hline
\end{tabular}

* Si veda la tabella 4.

giani. Il retroterra socio-professionale dei politici di carriera candidati nella $\mathrm{Ln}$ costituisce un interessante elemento di novità in quanto è piuttosto insolito che dalle professioni del lavoro autonomo e dell'imprenditoria provengano così tanti candidati con precedenti esperienze politiche. Non è da escludere che diversi, in questo gruppo di candidati, se eletti in parlamento, decidano di ritornare alla professione di origine dopo un solo mandato parlamentare, perché timorosi di rimanere troppo tempo lontani dalle loro attività di origine.

Anche in An è rilevante la presenza di candidati politici di carriera, con la forte presenza, tra le professioni liberali, degli avvocati $(25 \%)$ e quella dei quadri dirigenti ed intermedi del terziario privato. Complessivamente i candidati provenienti da tali categorie costituiscono il $50 \%$ del totale dei candidati politici di professione di An (Tab. 10). È interessante soffermarsi sull'elevata percentuale dei candidati provenienti dal terziario privato, che è la più alta registrata nelle tre liste della coalizione. Una possibile spiegazione di questa presenza imprevista e per certi aspetti sorprendente può essere data dal fatto che sul piano pro- 
fessionale i candidati politici di professione del terziario privato in An condividono con gli omologhi collocati nella Ln l'avversione nei confronti della burocrazia pubblica inefficiente e vessatoria e il risentimento per il trattamento, che considerano di favore, riservato agli addetti al pubblico impiego. Tali sentimenti, che nei candidati della $\mathrm{Ln}$ acquistano dimensione politica nel rifiuto del centralismo e della partitocrazia, nei candidati di An si saldano probabilmente con una identità ideologica forte, che può preesistere al momento dell'inizio dell'attività lavorativa e che si rafforza proprio per le caratteristiche del settore in questione e per la posizione lavorativa che essi vi occupano.

\section{L'azione dei gruppi di interesse}

Finora abbiamo esaminato la selezione dei candidati effettuata dai partiti che, però, potrebbero essere stati affiancati dalle associazioni di rappresentanza degli interessi economici in quanto anch'esse sono in grado, talvolta, di farsi veicolo di aggregazione della domanda di rappresentanza politica. Nel nostro caso, le associazioni che rappresentano $i$ grandi interessi economici organizzati, potrebbero essere state tentate di attivarsi durante l'ultima campagna elettorale approfittando dello spazio offerto dalla destrutturazione del sistema partitico.

In realtà, l'impegno delle associazioni di rappresentanza nella competizione elettorale, sia per numero di candidati presentati che per l'appoggio esplicito offerto alle singole formazioni politiche, è stato più limitato di quanto potesse far prevedere l'attivazione politica della società italiana avvenuta negli ultimi anni (campagne referendarie per la riforma del sistema elettorale, sostegno attivo all'azione della magistratura contro la corruzione politica, censura contro i corrotti, ecc.).

La mancata mobilitazione delle associazioni di interesse è, soprattutto, il risultato di una tendenza di lungo periodo contrassegnata dal progressivo declino dell'impegno diretto in politica dei gruppi di interesse. Una significativa presenza diretta dei rappresentanti dei gruppi di interesse nelle liste elettorali ed in parlamento si era manifestata in modo deciso dalla prima metà degli anni cinquanta, nel clima più generale della forte ideologizzazione dei rapporti tra i partiti e di questi con i gruppi, e delle opportunità offerte dal sistema elettorale proporzionale.

$\mathrm{Ma}$ in tempi più recenti le associazioni di rappresentanza 
sono state dissuase dal partecipare direttamente alle competizioni elettorali dalla crescente professionalizzazione del ceto parlamentare di estrazione partitica, che ha presidiato in misura sempre più vincolante le assemblee elettive scoraggiando accessi indipendenti. Inoltre, dall'inizio degli anni ottanta, diverse associazioni, e i sindacati in particolare, sono state investite da una crescente crisi di rappresentatività ${ }^{6}$ che ha sensibilmente attenuato le loro ambizioni politiche. Infine, non va dimenticato che le associazioni di interesse sono tutte organizzazioni «a legame debole» (Zan 1992), cioè con scarse capacità di azione unitaria finalizzata al conseguimento di uno scopo definito dalle leadership nazionali. Per di più, alcune associazioni sono ideologicamente divise al loro interno e ciò rende arduo impostare una strategia elettorale coerente.

Tutti questi fattori hanno contribuito a produrre lo scarso impegno delle associazioni di interesse e spiegano perché le candidature presentate nell'ultima campagna elettorale dai rappresentanti ufficili dei gruppi (pari al $4,5 \%$ dei candidati del nostro campione) siano state meno numerose delle previsioni. Alcune associazioni si sono, comunque, impegnate più di altre. Dalla tabella 11 emerge chiaramente che $\mathrm{i}$ sindacati sono stati $\mathrm{i}$ più attivi nel presentare candidature. Ciò era, del resto, prevedibile. I dirigenti sindacali sono, infatti, nella stragrande maggioranza, politici di professione che vantano precise affiliazioni partitiche anche perché la loro posizione al vertice dell'organizzazione sindacale è dovuta di solito alla sponsorship del partito di appartenenza. I sindacalisti possono, perciò, trovare posto tra i candidati più facilmente dei rappresentanti degli altri gruppi di interesse. Come in passato, anche nell'ultima campagna elettorale sono state le liste di sinistra ad ospitare il maggior numero di candidati dirigenti sindacali. In particolare, $i$ sindacalisti della Cgil si sono collocati nelle liste dei partiti che in passato hanno ricevuto la quota maggiore del voto operaio (Pds, Psi, Rc). Agli operai, ormai scomparsi dalle liste elettorali, i partiti storici dei lavoratori manuali hanno sostituito i loro rappresentanti nel mondo della produzione, per sottolineare la persistenza di un rapporto di identificazione con i problemi dei lavoratori che non si può più testimoniare in termini di rappresentatività sociologica. In particolare si è mossa in tale direzione Rc che

6 Sul punto cfr. Accornero 1992, 259-298. 
TAB. 11. I candidati dirigenti di associazioni di rappresentanza degli interessi economici. Valori assoluti

\begin{tabular}{|c|c|c|c|c|}
\hline \multirow[t]{2}{*}{ ASSOCIAZIONI } & \multicolumn{4}{|c|}{ COALIZIONI } \\
\hline & $\mathrm{Pgr}$ & $\mathrm{PpI}$ & Pdl-Pbg & Tot. \\
\hline $\begin{array}{l}\text { Cgil } \\
\text { Cisl } \\
\text { Uil } \\
\text { Cisnal } \\
\text { Sindacati }\end{array}$ & $\begin{array}{r}12 \\
4 \\
2 \\
4\end{array}$ & 5 & & $\begin{array}{r}12 \\
9 \\
2 \\
4 \\
27\end{array}$ \\
\hline $\begin{array}{l}\text { Confindustria } \\
\text { Confapi } \\
\text { Ass. industriali }\end{array}$ & 1 & 3 & $\begin{array}{l}4 \\
1\end{array}$ & $\begin{array}{l}8 \\
1 \\
9\end{array}$ \\
\hline $\begin{array}{l}\text { Confagricoltura } \\
\text { Coldiretti } \\
\text { Conf. coltivatori } \\
\text { Ass. agricole }\end{array}$ & 1 & $\begin{array}{l}2 \\
6 \\
1\end{array}$ & 2 & $\begin{array}{r}4 \\
6 \\
2 \\
12\end{array}$ \\
\hline Confcommercio & & & 1 & 1 \\
\hline $\begin{array}{l}\text { Confartigianato } \\
\text { Cna } \\
\text { Ass. artigiane }\end{array}$ & $\begin{array}{l}1 \\
1\end{array}$ & 3 & 1 & $\begin{array}{l}5 \\
1 \\
6\end{array}$ \\
\hline Totale & 22 & 20 & 13 & 55 \\
\hline
\end{tabular}

ha ospitato nelle sue liste 5 dei 12 sindacalisti della Cgil candidati della coalizione progressista. Una scelta analoga ha fatto anche Ottaviano Del Turco che ha cercato di mobilitare i sindacalisti socialisti della Cgil per agganciare il suo partito all'unico retroterra politico-sociale sul quale il Psi poteva ancora sperare di ricostituire la sua identità perduta. Ciò spiega anche la consistenza della risposta: i candidati sindacalisti provenienti dalla componente socialista della Cgil presentati dai progressiti nei collegi uninominali sono stati almeno 8 (ma il nostro campione ne comprende solo 3 ).

Una particolare menzione meritano i 4 sindacalisti di provenienza cislina che sono stati accolti nelle liste dei progressisti (Tab. 11). Costoro hanno aderito al gruppo dei cristiano-sociali di Carniti e Gorrieri che con quelle candidature ha voluto offrire un riferimento unitario al voto dei lavoratori cattolici e socialisti con l'intento di prefigurare il superamento delle divisioni esistenti tra le organizzazioni politiche e sindacali dei lavoratori. La maggioranza della Cisl ha, invece, appoggiato la coalizione 
di centro. I due candidati della Uil hanno aderito alle liste di Alleanza democratica. I 4 della Cisnal sono stati ospitati nelle liste di An.

Decisamente più contenuto di quello dei sindacalisti è stato il numero di candidati rappresentanti ufficiali degli industriali. Il dato può apparire sorprendente se confrontato con la presenza diretta degli imprenditori nelle liste, specie in quelle della coalizione di destra, ma si spiega con la posizione di equidistan$\mathrm{za}^{7}$ tra le coalizioni assunta dalla Confindustria. Tale posizione ha consentito alla leadership confindustriale di evitare una spaccatura formale dell'organizzazione nelle diverse opzioni emerse all'interno del mondo dell'industria nei mesi precedenti l'appuntamento elettorale. Le preferenze del presidente Abete e dei membri della giunta confindustriale a lui più vicini - almeno fino all'inizio del 1994 - andavano al Patto di Segni; un gruppo più cospicuo di dirigenti, che rifletteva l'orientamento della stragande maggioranza dei piccoli imprenditori associati alla confederazione, ha sostenuto, invece, la coalizione di destra, in particolare i candidati di Fi. Infine, una posizione di minoranza, espressa, però, da alcuni influenti industriali, è stata quella del cosiddetto «gruppo dei cento» che si è fatto promotore, appoggiando la lista di Alleanza nazionale, dell'idea di uno sviluppo economico che riesca a conciliare il sostegno alle imprese con la tutela dei diritti sociali. Ciascuna di queste differenti opzioni politiche si è concretata in alcune, poche, candidature di dirigenti confindustriali, che hanno, comunque, mostrato una maggiore preferenza per le liste collegate al Pdl-Pbg (Tab. 11). Senza esitazioni è stato il sostegno della Confapi a Fi e alla Ln a cui, però, come si vede dalla tabella 11 , non è seguito uno sforzo commisurato in termini di candidature.

Le associazioni degli agricoltori (con l'eccezione della Coldiretti), del commercio e dell'artigianato hanno assunto una posizione di attesa che, per lo più, è propria di organizzazioni con forte propensione filogovernativa, momentaneamente impedita dalla difficoltà di individuare i partiti e la coalizione che formeranno la maggioranza di governo. Sul piano operativo le organizzazioni in attesa hanno lasciato piena libertà d'iniziativa alle associazioni di primo grado ed ai singoli dirigenti locali. Le loro scelte non impegnavano le leadership nazionali, che si sono

\footnotetext{
7 Sui motivi, non solo congiunturali, che hanno indotto la leadership confederale ad assumere una posizione di equidistanza cfr. Mattina (1994, 155 s.).
} 
riservate di definire la collocazione politica dell'associazione dopo le elezioni. Le candidature, molto limitate, confermano la tradizionale difficoltà delle associazioni rappresentanti questi settori produttivi ad essere attive nell'arena elettorale ed indicano una preferenza per le liste di centro destra, con l'eccezione della Confcoltivatori e della Cna tradizionalmente collegate al Pds. Una considerazione a parte va fatta per la Coldiretti che, come è risaputo, in passato ha esibito straordinarie capacità di orientamento del voto dei piccoli conduttori agricoli sui propri candidati collocati nelle liste della Dc. Alla vigilia delle ultime elezioni la Coldiretti ha sostenuto il Ppi di Martinazzoli ma ha scoraggiato i propri dirigenti dall'accedere a cariche pubbliche stabilendo l'incompatibilità tra incarichi associativi e cariche parlamentari.

Le cifre esaminate inducono ad interrogarsi sugli sbocchi politici della spinta ad una maggiore partecipazione che si è registrata soprattutto tra i piccoli imprenditori, gli artigiani, insomma i ceti medi produttivi. Al quesito si è già in parte risposto nella prima parte di questo saggio, quando si è constatata la nuova rilevanza assunta dalla partecipazione diretta degli esponenti dei gruppi economici nelle liste elettorali. Ora, l'accertato, modesto, impegno delle associazioni di rappresentanza degli interessi economici, induce a ritenere che tale partecipazione diretta sia stata particolarmente cospicua. Su di essa punteremo la nostra attenzione nel prossimo paragrafo esaminando le dimensioni della presenza dei candidati senza precedenti politici e in quali direzioni essa si è indirizzata.

\section{L'accesso dei «non politici»}

Quali criteri i partiti hanno adottato per favorire il reclutamento dei candidati senza precedenti esperienze politiche? E con quali esiti? La risposta al secondo quesito in realtà l'abbiamo già anticipata nelle pagine precedenti individuando i politici di professione nel gruppo dei 601 che costituisce il 50\% del totale dei candidati del nostro campione. Dunque, l'altra metà dei candidati reclutati dai partiti è priva di esperienza politica; quantomeno ciò risulta dai dati raccolti. Naturalmente non si può escludere che diversi tra i candidati collocati tra i non politici in realtà siano politici di professione che fonti più affidabili dei profili biografici riportati dai quotidiani avrebbero consenti- 
to di individuare. A difesa dei profili biografici bisogna, comunque, dire che difficilmente il cronista esperto ed informato della vita politica locale avrebbe omesso di riportare un'informazione cruciale sul candidato come quella del suo eventuale impegno di consigliere comunale $o$, di più, di assessore o sindaco. D'altra parte dobbiamo anche supporre che lo stesso candidato, nel presentare la sua breve nota biografica, abbia di solito tenuto a sottolineare, e non a nascondere, la sua eventuale condizione di «esperto» della politica che lo collocava implicitamente in una posizione di vantaggio rispetto al candidato senza alcuna esperienza. Riteniamo, perciò, che i profili biografici abbiamo offerto in fin dei conti informazioni abbastanza accettabili per distinguere i due grandi ed equivalenti sottogruppi nei quali è stato possibile collocare i nostri candidati.

Il primo quesito richiede, invece, una risposta più articolata che può essere data solo attraverso l'esame delle differenti strategie seguite dalle tre coalizioni. Nella coalizione di destra ha prevalso il principio della rappresentatività e in subordine quello dell'appello alle professioni predisposte. Il $\mathrm{Pdl}-\mathrm{Pbg}$ ha confermato la sua preferenza per l'appello alle libere professioni, dalle quali ha reclutato il $36 \%$ dei suoi candidati senza precedenti esperienze politiche (Tab. 12) ${ }^{8}$. I liberi professionisti incidono infatti sul totale dei candidati «nuovi alla politica», presenti nelle liste del $\mathrm{Pdl}-\mathrm{Pbg}$, esattamente nella stessa misura rilevata per queste categorie calcolata sul totale delle figure professionali di tutti i candidati, con e senza esperienze politiche (Tab. 5), reclutati dalla coalizione di destra. Sostanzialmente confermata è risultata anche la quota di candidati provenienti dal mondo della scuola (istruzione superiore ed università).

Il Pdl-Pbg ha premuto, invece, l'acceleratore soprattutto nel settore del lavoro autonomo e delle attività imprenditoriali: $i$ candidati provenienti da queste aree di attività influiscono per il $35 \%$ sul totale dei candidati senza precedenti esperienze politiche (Tab. 12), cioè il $3 \%$ in più rispetto alla già elevata percentuale fatta registrare sul totale generale di tutti i candidati classificati per attività professionale, senza distinzione alcuna di

${ }^{8}$ Gli incroci tra i valori relativi alle professioni dei candidati e le variabili concernenti la presenza/assenza di eventuali esperienze politiche sono sempre stati effettuati su 1096 candidati: gli unici per i quali esistevano dati sufficienti ad effettuare tale rilevazione. Ciò spiega perché nella tabella 12 il numero di candidati senza esperienze politiche è pari a 551 unità. 
$\mathrm{T}_{\mathrm{AB}}$. 12. Le attività professionali dei candidati senza precedenti esperienze politiche (\%)

\begin{tabular}{|c|c|c|c|c|}
\hline \multirow[t]{2}{*}{ ASSOCIAZIONI } & \multicolumn{4}{|c|}{ COALIZIONI } \\
\hline & $\mathrm{Pgr}$ & PpI & Pdl-Pbg & Tot. \\
\hline Avvocati & 7 & 11 & 15 & 12,3 \\
\hline Medici & 12 & 14 & 14 & 13,2 \\
\hline Altri professionisti & 10 & 13 & 7 & 9,3 \\
\hline Insegnanti & 14 & 16 & 7 & 10,7 \\
\hline Docenti univ. & 15 & 11 & 3 & 7,6 \\
\hline Magistrati & 8 & 3 & 1 & 2,9 \\
\hline Imprenditori & 6 & 7 & 14 & 10,7 \\
\hline Dirigenti d'azienda & 1 & 5 & 5 & 4,2 \\
\hline Commerc. e artigiani & 2 & 1 & 6 & 3,4 \\
\hline Terziario privato* & 5 & 6 & 11 & 8,3 \\
\hline Giornalisti & 8 & 7 & 5 & 6 \\
\hline Dipendenti pubblici & 6 & 6 & 4 & 4,9 \\
\hline Pensionati & 3 & & 4 & 2,7 \\
\hline Altre attività & 4 & 2 & 3 & 2,2 \\
\hline $\begin{array}{l}\text { Totale } \\
\text { (N) }\end{array}$ & $\begin{array}{c}100 \\
(118)\end{array}$ & $\begin{array}{c}100 \\
(148)\end{array}$ & $\begin{array}{c}100 \\
(285)\end{array}$ & $\begin{array}{c}100 \\
(551)\end{array}$ \\
\hline
\end{tabular}

* Si veda la tabella 4.

curriculum politico. L'apertura verso queste figure appare particolarmente accentuata nella $\mathrm{Ln}$ e in $\mathrm{Fi}$, che da esse reclutano oltre il $40 \%$ del totale dei loro candidati senza precedenti esperienze politiche, con i significativi apici del $20 \%$ di addetti nel terziario all'interno della $\mathrm{Ln}$ e del $23 \%$ per gli imprenditori in $\mathrm{Fi}$ (Tab. 13). Rilevante è, anche, il fatto che An, pur rimanendo al di sotto delle percentuali delle altre due liste maggiori della coalizione di destra, recluta candidati senza precedenti esperienze politiche tra imprenditori e addetti al terziario in misura largamente superiore alle percentuali che possono vantare le liste delle due coalizioni concorrenti (Tab. 13).

Il Patto ha, invece, giocato pressocché esclusivamente la carta dell'appello alle professioni predisposte, accentuando l'apertura verso alcune figure professionali che erano già presenti in misura rilevante tra i suoi candidati e riducendo, al tempo stesso, la presenza in altre che più facilmente si qualificano come attività strumentali all'esercizio della politica come professione. Ha incrementato, infatti, la sua presenza tra le libere professioni e i docenti universitari, che insieme forniscono a questa coalizione il $65 \%$ dei candidati senza precedenti esperienze politiche, e ha ridotto il reclutamento di candidati dipen- 
TAB. 13. Le attività professionali dei candidati senza precedenti esperienze politiche, distinti per lista (\%)

\begin{tabular}{lrrrr}
\hline ATTIVITÀ & \multicolumn{3}{c}{ LISTE } \\
\cline { 2 - 5 } & Pds & Ln & Fi & An \\
\hline Avvocati & 4 & 16 & 9 & 18 \\
Medici & 12 & 10 & 19 & 12 \\
Altri professionisti & 4 & 10 & 4 & 4 \\
Insegnanti & 12 & 10 & 7 & 6 \\
Docenti univ. & 20 & 5 & 3 & 2 \\
Magistrati & 12 & 7 & 2 & 1 \\
Imprenditori & & 8 & 8 & 11 \\
Dirigenti d'azienda & 4 & 8 & 3 & 2 \\
Commerc. e artigiani & 4 & 20 & 8 & 5 \\
Terziario privato & 4 & 3 & 5 & 12 \\
Giornalisti & 12 & 3 & 3 & 7 \\
Dipendenti pubblici & 8 & & 2 & 8 \\
Pensionati & 4 & & 4 & 6 \\
Altre attività & 4 & 100 & 100 & 100 \\
& 100 & $(61)$ & $(91)$ & $(107)$ \\
Totale & $(25)$ & & & \\
(N) & & & & \\
\hline
\end{tabular}

denti pubblici ed insegnanti, che rimangono, però, rilevanti sul totale dei candidati senza curriculum politico (Tab. 12). Gli insegnanti, in particolare, mantengono alta un'offerta di candidati che, molto presumiblmente, è resa possibile da un certo radicamento dell'associazionismo cattolico nel mondo della scuola. Sostanzialmente inalterata rimane, invece, la quota di candidati reclutati nel lavoro autonomo e tra gli imprenditori, a testimonianza del fatto che in queste categorie la coalizione di centro non appare in grado di acquisire adesioni oltre un certo limite $(19 \%)$.

La sinistra ha aperto il reclutamento ai candidati senza curriculum politico cercando di irrobustire la sue liste della presenza di quelle categorie di candidati che in generale sono risultate le meno rappresentate a causa della forte incidenza dei politici di professione nella coalizione progressista. I progressisti hanno, perciò, seguito sia il criterio dell'appello alle professioni predisposte per incrementare il numero di candidati provenienti dai liberi professionisti, che quello della rappresentatività con l'obiettivo di reclutare più addetti dal lavoro autonomo e dagli imprenditori. Di conseguenza, rispetto al totale dei candidati liberi professionisti presenti nelle liste di sinistra, quelli senza curricula politici passano dal $20,7 \%$ al $29 \%$; invece i candidati 
provenienti dai ceti medi produttivi raddoppiano quasi la loro presenza, passando dal $7,5 \%$ al $14 \%$ (Tab. 12). Sostanzialmente allo stesso livello del totale generale si colloca la quota di candidati senza curricula politici provenienti dal mondo della scuola, dove la presenza dei candidati della sinistra è risultata particolarmente forte. Tra i candidati privi di curricula politici aumenta anche la quota di quelli provenienti dalla magistratura e dal giornalismo. Il risultato della strategia dell'adozione del doppio criterio è che nelle libere professioni i candidati della sinistra senza precedenti politici si appaiano a quelli della destra $(29 \%)$, mentre nel terziario e nel lavoro autonomo rimangono comunque distanti dai candidati della destra.

Per ricapitolare, il Patto per l'Italia e il Pdl-Pbg mantengono o migliorano le posizioni «forti» accertate sul totale di tutti i candidati, mentre la sinistra sembra aver compiuto uno sforzo significativo per incrementare il numero dei suoi candidati nei settori meno rappresentati all'interno delle sue liste. Nel complesso queste diverse strategie non contribuiscono, tuttavia, ad attenuare la specificità settoriale dei bacini di reclutamento in cui ciascuna delle tre coalizioni ha attinto il maggior numero dei suoi candidati. Infatti, né il Patto né tantomeno i progressisti riescono a «sfondare» nel settore del lavoro autonomo saldamente presidiato dalla destra. Questa, a sua volta, non pare interessata, o capace, di incrementare il numero dei suoi candidati provenienti dal mondo dell'istruzione, che rimane un bacino privilegiato di reclutamento della sinistra e del Patto. Per quanto riguarda le libere professioni esse forniscono quasi un terzo dei candidati senza precedenti esperienze politiche a due coalizioni e ben il 38\% alla terza: contribuiscono, in tal modo, ma in misura molto limitata, ad attenuare le differenze nella composizione delle liste dei candidati nelle coalizioni concorrenti.

A prescindere dai risultati diseguali che ciascuna coalizione e le liste ad essa collegate hanno ottenuto, non si può negare che uno sforzo i partiti lo hanno compiuto per migliorare la ricettività dei canali della rappresentanza politica. E lo hanno fatto aprendo le porte a candidati provenienti dalla società che non sono stati scelti in funzione ornamentale o di rincalzo dei candidati già dotati di precedenti esperienze politiche; del resto la logica inesorabile dei collegi uninominali non consentiva scelte strumentali. Infatti, i candidati senza curricula politici hanno mostrato di essere complessivamente molto competitivi (Tab. 14) rispetto ai candidati con precedenti esperienze che pur disponevano di maggiori risorse politiche. 
TAB. 14. I vincitori, e i piazzati, nei 374 collegi uninominali del campione, distinti tra candidati con e senza precedenti esperienze politiche (1.096 casi)

\begin{tabular}{lrrrrr}
\hline & \multicolumn{2}{c}{ Esp. pol } & & \multicolumn{2}{c}{ Senza esp. pol. } \\
\cline { 2 - 3 } \cline { 5 - 6 } & $\mathrm{N}$ & $\%$ & & $\mathrm{~N}$ & $\%$ \\
\hline Vincitori & 193 & 17,6 & & 140 & 12,8 \\
Secondi & 144 & 13,2 & & 150 & 13,7 \\
Terzi & 135 & 12,3 & & 155 & 14,2 \\
Oltre il 30 posto & 73 & 6,6 & & 106 & 9,6 \\
Totale & 545 & 49,7 & & 551 & 50,3 \\
\hline
\end{tabular}

\section{Conclusioni}

Concludiamo con una considerazione sul rapporto partitielettori che scaturisce dall'analisi sulle candidature. Si può dire che le candidature hanno finito per riflettere in modo trasparente le fratture lungo le quali si è diviso l'elettorato durante la campagna elettorale e che si sono riprodotte tra le coalizioni non solo nella contrapposizione dei programmi ma in qualche misura anche nelle caratteristiche socio-politiche dei candidati collegati alle diverse liste.

I profili dei candidati che abbiamo ricostruito consentono, infatti, di collocarli abbastanza agevolmente sui lati opposti delle principali linee di frattura sulle quali si è diviso l'elettorato: il conflitto sulla preferenza da accordare alla dimensione pubblica o a quella privata nell'azione di governo e quello della priorità da dare alla tutela del lavoro dipendente o al sostegno al lavoro autonomo. Queste fratture durante la campagna elettorale si sono concretate con l'opzione «pubblico-lavoro dipendente» da un lato contrapposta a «privato-lavoro autonomo» dall'altro, con una variante «territoriale» delle due rappresentata dalle leghe che hanno contrapposto «federalismo» a «centralismo».

Ebbene, come abbiamo visto dalla nostra analisi, la distinzione più rilevante tra coalizione di sinistra e di destra dal punto di vista delle attività professionali dei candidati è passata proprio lungo la linea di divisione del rapporto tra pubblico e privato, e tra lavoro autonomo e lavoro dipendente. La coalizione progressista, caratterizzata per la forte presenza dei politici di professione e per il massiccio insediamento nel pubblico impiego, ci appare, perciò, come la fedele raffigurazione socio-politica degli interessi e dei gruppi sociali che si sono riconosciuti 
nella prima opzione. Sull'altro versante il $\mathrm{Pdl}-\mathrm{Bg}$, ed in particolare Fi e la Ln, con la forte presenza di candidati provenienti dalle libere professioni, dalle attività imprenditoriali e dal lavoro autonomo, si qualificano concretamente per quanto hanno detto nei loro programmi, cioè per i fautori dello stato minimo e di una legislazione poco vincolistica del mercato del lavoro.

Nel mezzo, tra destra e sinistra, i candidati del Ppi raffigurano tangibilmente la contraddittoria posizione politica della coalizione cui appartengono. I candidati del centro appaiono, infatti, divisi tra la componente - maggioritaria - proveniente dal pubblico impiego, affine per ambiente sociale di provenien-

TAB. 15. Distribuzione ponderata dei candidati delle tre coalizioni su quattro settori lavorativi (\%)

\begin{tabular}{lcccc}
\hline & Pgr & PpI & Pdl-bg & Tot. \\
\hline Professionisti & 22,4 & 37,4 & 40,2 & 100 \\
Lavoro autonomo & 12,2 & 31,8 & 56 & 100 \\
Pubblico impiego & 41,3 & 39,3 & 19,4 & 100 \\
Politici & 69,2 & 15,4 & 15,4 & 100 \\
\hline
\end{tabular}

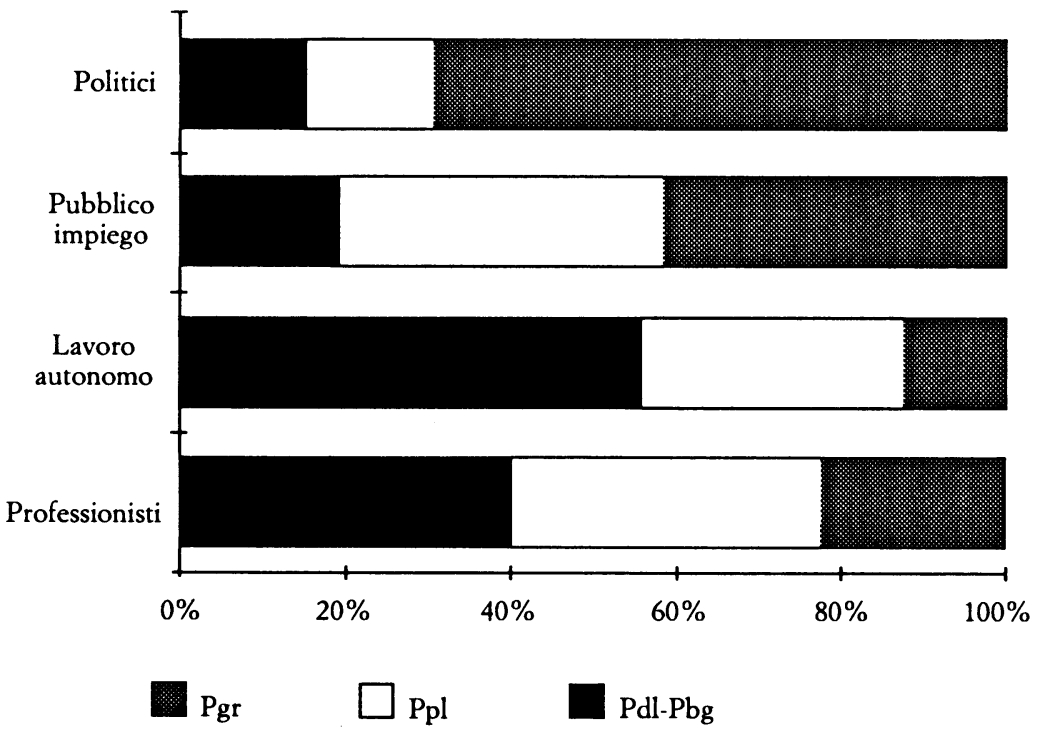

FIG. 1. Candidati distinti per settori lavorativi omogenei 
za e interessi rappresentati ai candidati della sinistra, e la componente dei liberi professionisti più vicina alle idee e aspettative rappresentate dai candidati della destra.

Le linee di frattura che hanno diviso l'elettorato risultano, dunque, molto nettamente riprodotte nella composizione delle candidature. Ciò appare con evidenza nella distribuzione ponderata dei candidati delle tre coalizioni sulle principali quattro aree di occupazione a cui si possono ridurre le loro diverse attività professionali (Tab. 15 e grafico). Naturalmente è lecito supporre che alcune formazioni abbiano voluto sottolineare le fratture esistenti, e le opzioni da loro proposte, selezionando candidati che hanno sovrarappresentato la presenza di certi gruppi sociali o professioni; sicuramente in questi termini va interpretata la forte presenza degli imprenditori nelle file di Fi, quella dei sindacalisti in Rc e nel Psi, o quella dei lavoratori autonomi nella $\mathrm{Ln}$. D'altra parte è anche probabile che quei candidati abbiano finito per riflettere l'attuale insediamento sociale dei partiti che li hanno selezionati o l'estensione dell'ombrello (e del progetto) politico sotto il quale i leaders politici delle diverse coalizioni ritenevano fosse possibile raccogliere la quota maggioritaria dell'elettorato. Se così è, l'analisi che abbiamo condotto sui candidati può offrire utile materia di riflessione a vincitori e vinti.

\section{Riferimenti bibliografici}

Accornero, A. (1992), La parabola del sindacato, Bologna, Il Mulino. Censis (1994), Vecchi politici o nuovi notabili. Radiografia dei candida$t i$, Roma.

Cotta M. (1979), Classe politica e parlamento in Italia: 1946-1976, Bologna, Il Mulino.

Di Palma, G. e M. Cotta (1986), Cadres, Peones, and Entrepreneurs: Professional Identities in a Divided Parliement, in E. Suleiman (a cura di), Parliaments and Parliamentarians in Democratic Politics, New York, Holmes and Meier, pp. 41-78.

Mastropaolo, A. (1993), Il ceto politico. Teoria e pratiche, Roma, La Nuova Italia.

Mattina, L. (1994), Gli attori economici. Vincitori e vinti, in I. Diamanti e R. Mannheimer, Milano a Roma. Guida all'Italia elettorale del 1994, Roma, Donzelli.

Sartori, G. (1987a), Rappresentanza, in G. Sartori, Elementi di teoria politica, Bologna, Il Mulino, pp. 269-310. 
- (1987b), Parlamento, in G. Sartori, Elementi di teoria politica, Bologna, Il Mulino, pp. 201-240.

Suleiman, E. (1986), Towards the Disciplining of Parties and Legislators: The French Parlamentarian in the Fifth Republic, in E. Suleiman (a cura di), Parliaments and Parliamentarians in Democratic Politics, New York, Holmes and Meier, pp. 79-106.

Zan, S. (1992), Organizzazione e rappresentanza. Le associazioni imprenditoriali e sindacali, Roma, La Nuova Italia. 